Division of Geological \& Geophysical Surveys

PRELIMINARY INTERPRETIVE REPORT 2005-1

\title{
Bristol Bay and Alaska Peninsula 2004: Fieldwork and Sample Analyses Compilation Report
}

\author{
by
}

Rocky R. Reifenstuhl, Rebecca D. Bailey, and Emily S. Finzel

February 2005

THIS REPORT HAS NOT BEEN REVIEWED FOR TECHNICAL CONTENT (EXCEPT AS NOTED IN TEXT) OR FOR CONFORMITY TO THE EDITORIAL STANDARDS OF DGGS.

Released by

STATE OF ALASKA

DEPARTMENT OF NATURAL RESOURCES

Division of Geological \& Geophysical Surveys

3354 College Rd.

Fairbanks, Alaska 99709-3707

$\$ 2.00$ 


\section{Table of Contents}

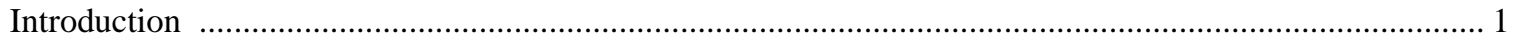

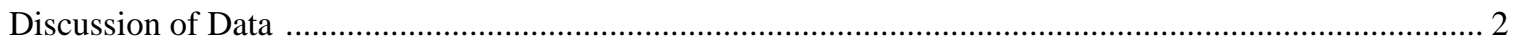



Figure 2: Sample Location Map, Mesozoic Section, Puale Bay and Wide Bay areas .............................. 5

Figure 3: Sample Location Map, Tertiary Section, Port Moller, Herendeen Bay,

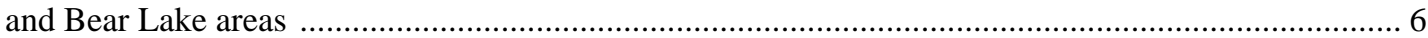

Figure 4: Generalized Mesozoic Stratigraphic Section.................................................................... 7

Figure 5: Generalized Tertiary Stratigraphic Section ................................................................. 8

Table 1: List of Sample Locations and Analyses Performed ..........................................................

Table 2: Headspace Gas Analysis of Gas Seep Sample ................................................................

Table 3a: Whole Oil Geochemical Analysis of Oil Seep Sample -

Parameters used in whole oil geochemical analysis ............................................................... 10

Table 3b: Whole Oil Geochemical Analysis of Oil Seep Sample -



Table 3c: Whole Oil Geochemical Analysis of Oil Seep Sample -

Whole oil geochemical analysis results

Table 3d: Whole Oil Geochemical Analysis of Oil Seep Sample

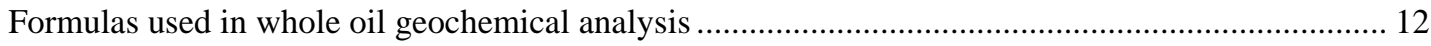

Table 4: Total Organic Carbon, Pyrolysis Analysis of 15 Outcrop Samples .......................................... 13

Figure 6: Kerogen Type Determination of Total Organic Carbon Outcrop Samples ................................. 14

Table 5: Porosity and Permeability Analyses of 81 Outcrop Samples............................................... 15

Figure 7: Porosity and Permeability of Outcrop Samples (undifferentiated)....................................... 16

Figure 8: Porosity and Permeability of Outcrop Samples (by formation) ........................................... 17

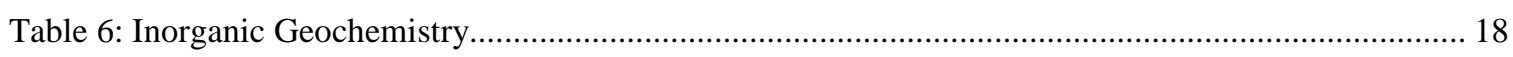

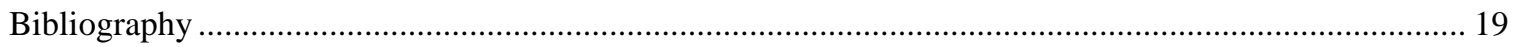




\title{
Bristol Bay and Alaska Peninsula 2004: Fieldwork and Sample Analyses Compilation Report
}

\author{
by Rocky R. Reifenstuhl (rocky_reifenstuhl@dnr.state.ak.us), Rebecca D. Bailey, and Emily S. Finzel
}

\section{Introduction}

This preliminary report is based on the 2004, twenty-two day, two-phase, Bristol Bay-Alaska Peninsula field program (Figure 1). The program's first phase focused on source rock potential of the Mesozoic section (Figure 2; Figure 4). The second phase focused on reservoir potential and stratigraphic architecture of Tertiary rocks (Figure 3; Figure 5). Data included here are rock sample details and field station locations, headspace gas analyses, whole oil geochemistry, organic geochemistry, total organic carbon, kerogen typing, porosity and permeability, and inorganic geochemistry. Separate reports from the Alaska Division of Geological \& Geophysical Surveys (DGGS) summarize the megafossil data (Blodgett, in preparation) and the measured stratigraphic sections (Ridgway and Finzel, in preparation). Both bear on surface to subsurface stratigraphic correlations.

This report is relevant to hydrocarbon-resources and exploration models within the state Division of Oil and Gas's Bristol Bay area-wide lease sales for 2005 and beyond, and is available on the DGGS website (http://www.dggs.dnr.state.ak.us). The Division of Oil and Gas (DOG) website (http://www.dog.dnr.state.ak.us) includes a variety of Bristol Bay and Alaska Peninsula information including geology, well locations and details, cross section, area-wide lease sale maps and information, and public seismic data.

Field-based results presented here are part of the Bristol Bay basin petroleum reservoir characterization, source rock potential, and fossil fuel resources program (2004-2007), funded by the U.S. Department of Energy through the Arctic Energy Technology Development Laboratory at the University of Alaska Fairbanks, DGGS, and DOG. Geologists participating are: Rocky Reifenstuhl (DGGS; program head), Dave Shafer (DOG), Tim Ryherd (DOG), Don Brizzolara (DOG), Robert Blodgett (consulting megafossil paleontologist), Mark Myers (DOG), Emily Finzel (DGGS), Ken Ridgway (Purdue University), and Paul McCarthy (University of Alaska Fairbanks; non-field collaborator). The manuscript was reviewed by Marwan Wartes of DGGS.

The first phase of this project included 11 days (May 24 to June 4, 2004) of helicopter-supported fieldwork in the Puale Bay and Wide Bay areas, northeast Alaska Peninsula (Figures 1 and 2). Base camp facilities were at Grizzly Skins Lodge near the Kajulik River and Becharof Lake. Our primary goal for this field investigation was to better understand the source rock potential and stratigraphy of the Mesozoic (Jurassic and Triassic) section (Figure 4), and sample oil seeps for geochemical characterization.

The second phase was 11 days (August 23 to September 2, 2004) of helicopter-supported fieldwork in the Port Moller, Herendeen Bay, and Bear Lake area, Bristol Bay (Figures 1 and 3). Base camp facilities were at Bear Lake Lodge on Bear Lake. This portion of the project focused on the reservoir potential of the Tertiary strata (Figure 5), particularly the Miocene Bear Lake and Pliocene Milky River formations.

Outcrop field stations and samples are all from the Mesozoic and Tertiary part of the stratigraphic section (Figures 4 and 5). Mesozoic samples from the northeast side of the Alaska Peninsula are from the Kamishak (Triassic), Talkeetna (Lower Jurassic), Kialagvik (Middle Jurassic), Shelikof (Middle Jurassic), and Naknek (Upper Jurassic) formations. Mesozoic samples from the greater Port Moller area are from the Herendeen Limestone (Lower Cretaceous) and the Coal Valley Member (Upper Cretaceous) of the Chignik Formation. Tertiary outcrops in the greater Port Moller area include the Tolstoi (Eocene), Stepovak (Oligocene), Bear Lake (Miocene), and Milky River (Pliocene) Formations.

This report is intended as a timely public release of a wide variety of analytical data on outcrop samples collected in the first field season of this project. Each of the tables in this report is listed below with some pertinent background information. 


\section{Discussion of Data}

Table 1 - List of Sample Locations and Analyses Performed: This is a compilation of basic field station information, including latitude and longitude, rock unit, and the analysis performed.

Table 2 - Headspace Gas Analysis of Gas Seep Sample: Results from one gas sample (04RR139A) from the hot springs in Herendeen Bay, southwest of Port Moller (139 on Figure 3). Results indicate more than 99 percent methane. Gas bubbles strongly through the hot springs pool, which is located in a vegetated point of land on the west side of Port Moller Bay. Scheduled for the 2005 field season is a sampling program that will collect additional gas for isotopic analyses aimed at constraining the source of the gas generation.

Tables 3a through 3d - Whole Oil Geochemical Analyses of Oil Seep Sample: Whole oil analyses are from one sample of the oil and gas seep near Oil Creek (04RR14; 14 on Figure 2). Detailed analyses indicate a highly biodegraded oil. The seep flows an estimated one-half barrel per day into a small pool of water in a tundra and muskeg area some 100 meters from the main Oil Creek waters. Most of the light hydrocarbons evaporate prior to the oil seep reaching Oil Creek. A 150-meter-wide delta-shaped fan has been deposited over time; it consists of extremely viscous, tarry hydrocarbon. To the southeast and downstream 500 to 700 meters on Oil Creek, very small quantities of hydrocarbon flow directly from fractures in the very fine sandstone of the Shelikof Formation. These minor seeps produce an oily sheen on some creek waters. Locally, there are minor occurrences in the outcrops of sandstone that have a dark opaque matrix that appear to be oil charged. Importantly, the hydrocarbon typing analyses of the oil seep sample from Oil Creek is in part correlative with the Kamishak Formation source rock analyses.

Table 4 - Total Organic Carbon, Pyrolysis Analysis of 15 Outcrop Samples: Rocks analyzed range from the Triassic Kamishak Formation from the northeast point of land of Puale Bay to the Miocene Bear Lake Formation from Port Moller. One Kamishak Formation limestone yields 2.38 percent total organic carbon (TOC). This rock is from a carbonate section that includes biohermal deposits. A similar muddy limestone from the same Kamishak Formation section yields 1.12 percent TOC. More detailed source rock sampling of the Kamishak Formation will be conducted during the 2005 field program. The Middle Jurassic Kialagvik Formation samples (3) range from 0.54 to 0.81 percent TOC. The Kialagvik Formation also will be sampled systematically during the 2005 field program. One sample was collected from the Snug Harbor Member of the Naknek Formation (Upper Jurassic) and yields 2.19 percent TOC. This locality is 1 kilometer west of the southwest arm of Becharof Lake. Based on the silty claystone and shale lithologies of this section, which yielded relatively high TOC, additional sampling is scheduled during the 2005 season. The Oligocene Stepovak Formation sample is from a section southeast of Bear Lake, and consists of interbedded sandstone, siliceous siltstone and black coaly material. The sample yields 9.54 percent TOC. The high carbon content is due to coaly fragments in the sediment. A back-bay or lagoonal depositional environment is interpreted for these sediments. The carbon content in the Stepovak Formation and Bear Lake Formation samples ( 0.21 and 0.08 percent TOC), combined with low hydrogen index values, suggest that the kerogen is most likely gas prone woody material. The remaining samples in the table are from marine or deep marine depositional environments (see Figure 1 for kerogen typing analyses of 12 samples). More detailed sampling and analyses will more closely constrain kerogen typing during 2005.

Figure 6 - Kerogen Type Determination, 2004 Bristol Bay outcrop samples: Shows the hydrocarbon generating kerogen from 12 selected rocks. The background discussion of the rock samples and their formations are in Table 4 notes above. The three oil-prone samples on the graph are from the Kamishak Formation and from oil-charged sandstone from the Snug Harbor Member of the Naknek Formation. Importantly, the hydrocarbon typing analyses of the oil seep sample from Oil Creek (Table 3) is in part correlative with the Kamishak Formation source rock analyses.

Table 5 - Porosity and Permeability Analyses of 81 Outcrop Samples: This table is a list, arranged from youngest to oldest, of the nine rock units which range from Pliocene to Middle Jurassic; all are outcrop samples.

Figure 7 - Porosity and Permeability of Outcrop Samples (undifferentiated): This is a plot showing porosity and permeability results for 59 outcrop samples. Approximately half of the samples are from the Mesozoic section. Mesozoic samples are uniformly low in both porosity and permeability, with the exception of four samples from the Chisik Member of the lower Naknek Formation. These Mesozoic rock units were sampled in an effort to obtain a 
more complete petrophysical outline of the entire stratigraphic succession on the Alaska Peninsula. The majority of the sampled intervals were never considered to be potential reservoir targets. Rather, they were the focus of source rock potential or depositional environment studies, and outcrop petrophysics samples were collected for comprehensiveness. Consequently, the porosity and permeability values are quite low for two of the three Middle and Late Jurassic units (Kialagvik Formation, Shelikof Formation, and for some of the Naknek Formation). When considering all samples, only 10 percent have more than 1 millidarcy permeability, and these have corresponding porosity values ranging from 5 to 37 percent. About 30 percent of the 81 samples have permeability greater than 0.10 millidarcy, with porosity in the 5 to 15 percent range. Despite poor permeability $(<0.10$ millidarcy), these rocks can be considered viable gas reservoirs. It's important to note that several outcrops of the Chisik Conglomerate Member of the basal Naknek Formation, the Bear Lake Formation, and the Milky River Formation were too unconsolidated to process for petrophysical analyses. Considering that the Bear Lake Formation is expected to be the reservoir in many published hydrocarbon play models, this is an important aspect that the data alone do not portray.

Figure 8 - Porosity and Permeability of Outcrop Samples (by formation): This plot summarizes the petrophysical analyses for the nine rock units that range from Pliocene to Middle Jurassic. The Pliocene Milky River Formation samples from the Port Moller area have the best reservoir quality, followed by the Chisik Member of the Naknek Formation, and the Bear Lake Formation. Several samples plotted from the Bear Lake, Milky River, and Chisik Conglomerate formations do not convey that several outcrops of these units were too unconsolidated for obtaining core samples. That aside, the vast majority of Bear Lake samples have good porosity but their corresponding permeability is low to poor. Petrographically, the low permeability is apparently due to alteration products of volcanic rock fragments and pore-clogging clays. These diagenetic processes have reduced the pore connectivity in many samples. Milky River Formation sample values are the best with a maximum of 3,500 millidarcies and 32 percent porosity. The Pliocene Milky River Formation is relatively high in the stratigraphic section, and in many published hydrocarbon play models, is considered a less likely reservoir than the underlying Miocene Bear Lake Formation.

Table 6 - Inorganic Geochemistry: Analyses of one sample (04RR20C) of the Upper Cretaceous Chisik Conglomerate Member of the Naknek Formation reveals that this dense, magnetic, oil-charged sandstone contains 23 percent, by weight, magnetic minerals. The Chisik Member on the southeast side of Deer Mountain, northwest of Wide Bay (Figure 2), is a succession of sandstone and conglomerate. The channelized, non marine- to marginalmarine, very-coarse grained sandstone is very dark gray to black, poorly sorted, with subrounded grains, and has a slightly greasy feel. The sandstone is nearly 17 percent iron $\left(\mathrm{Fe}_{2} \mathrm{O}_{3}+\mathrm{FeO}\right)$, with only 1.2 percent titanium oxide, suggesting that magnetite, not ilmenite, is the dominant magnetic mineral. The same sandstone has 13.9 percent porosity and 0.22 millidarcy permeability. The concentrated magnetic minerals were likely derived from the intermediate volcanic and plutonic rocks of the Jurassic arc system. Abundant plutonic and volcanic cobbles in the interbedded conglomerate support this interpretation.

This preliminary report is significant in that it documents oil-charged sandstone outcrops in the Chisik Member of the lower Naknek Formation on the east side of Deer Mountain, and south of Puale Bay on the ridge northeast of Teressa Creek (04RR20C; number 20 on Figure 2). Our goal with this raw data release is to inform the energy industry and general public of new hydrocarbon exploration-pertinent information for the Mesozoic section in the study area.

Field observations and organic geochemical analyses yield new, modern information that bear on Mesozoic reservoir characteristics, stratigraphy, source rock total organic carbon (TOC), liquid hydrocarbon typing from a seep between Oil Creek and Ugashik Creek (Figure 2), and distribution of stratigraphic units in the Puale Bay and Wide Bay areas. This ongoing Bristol Bay-Alaska Peninsula program is scheduled for additional fieldwork at the end of August 2005 in the Bear Lake area, northeast of Port Moller. During these two weeks we will address the reservoir potential, stratigraphic architecture, and structural framework of the Tertiary stratigraphic section. These Tertiary rocks, particularly the Miocene Bear Lake Formation, are potential reservoirs in the Bristol Bay region. 


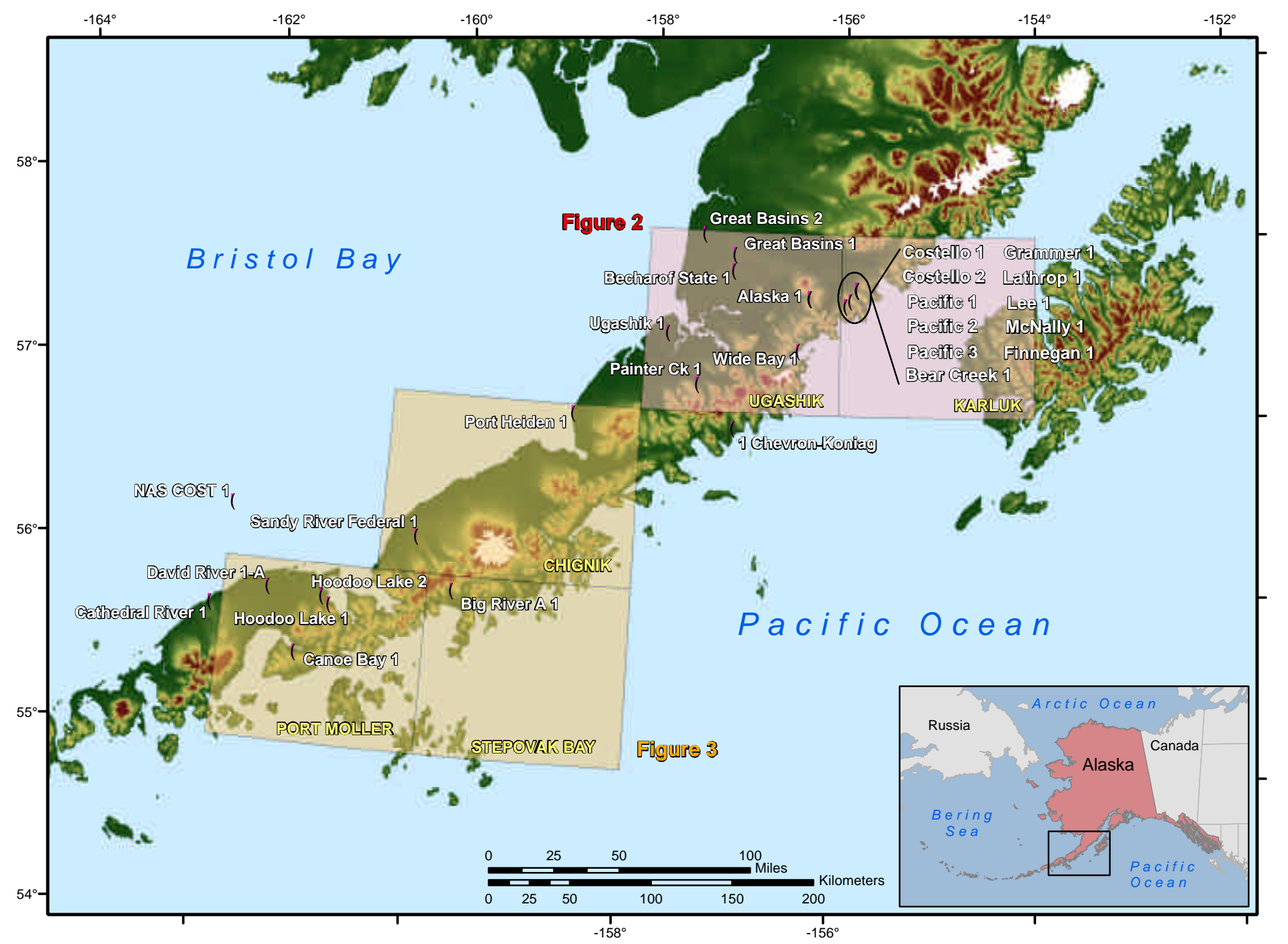

Figure 1. Field area location map of Alaska Peninsula and Bristol Bay, showing locations of figures 2 and 3, wells drilled in the area, and quadrangle index. 


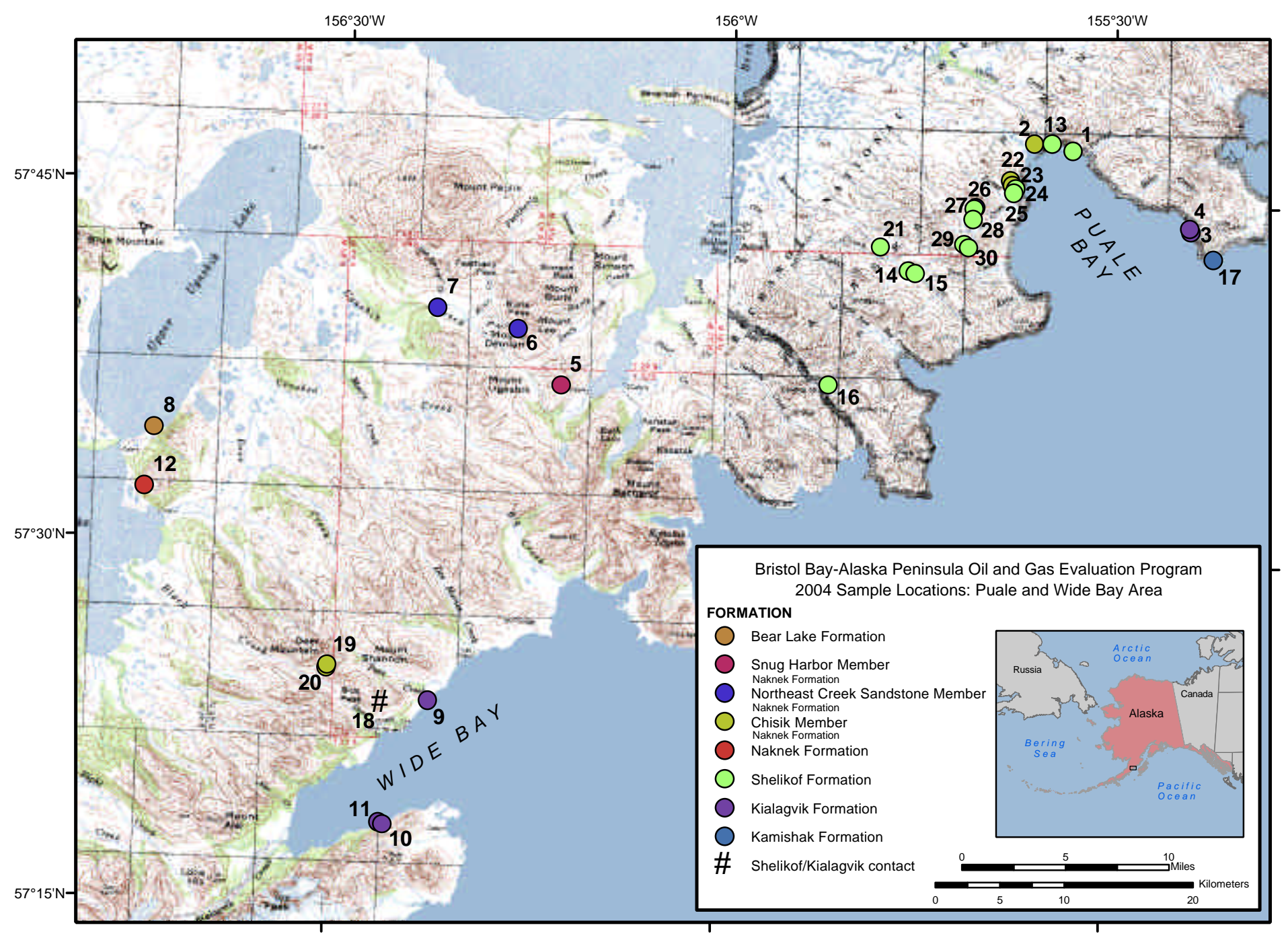

Figure 2. Location map for Rocky Reifenstuhl's sample locations, Phase 1, Mesozoic section. Labels are the last two digits of sample identifications listed in the text and tables. 

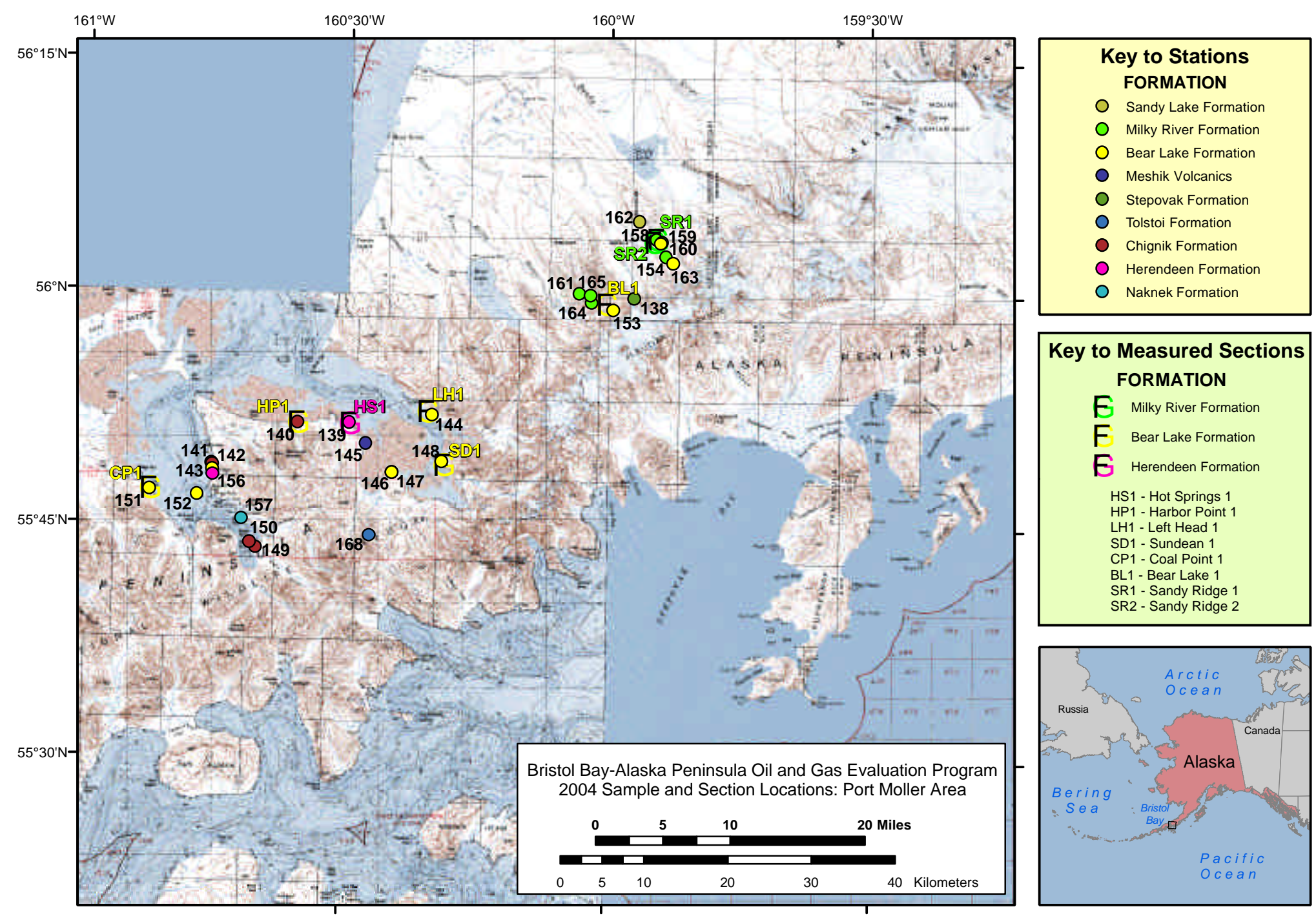

Figure 3. Location map for Rocky Reifenstuhl's sample locations, Phase 2, Tertiary section. Labels are the last two digits of sample identifications listed in the text and tables, or abbreviations for measured sections. 




\section{Exposed section near Puale and Wide bays}

Oil stains in Cathedral River \#1, dead oil and stain in Koniag \#1

Oil stains in Cathedral River \#1

Oil staining in Bear Creek \#1, Wide Bay \#1 (cores), Grammer \#1, Cathedral River \#1

Dead oil in Bear Creek \#1, oil stains in Wide Bay \#1, Cathedral River \#1

Solid hydrocarbons in Bear Creek \#1 (Kamishak Fm.)

(O) = Stratigraphic position of oil shows reported in well logs

Modified from Detterman and others, 1996

Figure 4. Generalized Mesozoic stratigraphic section in the Alaska Peninsula and Bristol Bay area. Circles indicate stratigraphic position of oil shows reported in well logs and literature. 




Figure 5. Generalized Tertiary stratigraphic section in the Alaska Peninsula and Bristol Bay area. From Detterman and others, 1996. 


\begin{tabular}{|c|c|c|c|c|c|c|c|c|c|c|}
\hline \multicolumn{3}{|c|}{ Sample ID FormationAnalysis LatitudeLongitude } & \multirow{2}{*}{\begin{tabular}{|l|} 
Sample ID \\
04RR138
\end{tabular}} & \multicolumn{3}{|c|}{ FormationAnalysis LatitudeLongitude } & \multicolumn{4}{|c|}{ Sample IDFormationAnalysis LatitudeLongitude } \\
\hline \multicolumn{2}{|c|}{ 04RR154B Milky RiverPP } & 56.04404-159.89347 & & Stepovak & $\overline{P P P}$ & $255.99835-159.95333$ & 04RR7B & Naknek & $\overline{P P P}$ & $57.66677-156.37852$ \\
\hline \multicolumn{2}{|c|}{ 04RR158CMilky RiverPP } & $55.06233-159.91165$ & O4RR138D & Stepovak & TOC & $55.99835-159.95333$ & 04RR7D & Naknek & $\mathrm{PP}$ & 56.37852 \\
\hline \multicolumn{2}{|c|}{ 04RR158GMilky RiverPP } & 55.06233-159.91165 & 04RR155B & Tolstoi & PP & $55.48712-161.47603$ & 04RR7B & Naknek & PP & $57.66677-156.37852$ \\
\hline SR2-6 & Milky RiverPP & $56.06010-159.91490$ & 04RR155B & Tolstoi & $\mathrm{PP}$ & $55.48712-161.47603$ & 04RR7D & Naknek & $\mathrm{PP}$ & $57.66677-156.37852$ \\
\hline SR2-89.5 & Milky RiverPP & $56.06010-159.91490$ & O4RR168B & Tolstoi & $\mathrm{PP}$ & $55.74076-160.45229$ & 04TJR09 & Naknek & $\mathrm{PP}$ & $57.53558-156.75181$ \\
\hline SR1-3.5 & Milky RiverPP & $56.06151-159.91139$ & 04RR141B & Chignik & $\mathrm{PP}$ & $55.81506-160.75445$ & 04TJR4G & Naknek & TOC & $57.61553-$ \\
\hline \multicolumn{2}{|c|}{ 04RR140B Bear Lake TOC } & 55.8 & $142 A$ & Chignik & TOC & 55.8 & 04DS10A & Shelikof & TOC & 57119 \\
\hline \multicolumn{2}{|c|}{ 04RR140CBear Lake PP } & $55.86046-160.59232$ & O4RR142B & Chignik & PP & $55.81390-160.75302$ & O4DS10B & Shelikof & TOC & $57.79258-155.57110$ \\
\hline \multicolumn{2}{|c|}{ O4RR140E Bear Lake TOC } & $55.86046-160.59232$ & O4RR149B & Chignik & PP & $55.72546-160.66745$ & 04DS11 & Shelikof & TOC & 55.86644 \\
\hline \multicolumn{2}{|c|}{ 04RR148B Bear Lake PP } & 55.8 & O4RR150B & Chi & $\mathrm{PP}$ & $55.73109-$ & R13B & elikof & $\mathrm{PP}$ & 8232 \\
\hline \multicolumn{2}{|c|}{ 04RR152CBear Lake PP } & 55.7 & 04RR151C & Chignik & PP & 55.7 & $313 C$ & Shelikof & TOC & 8232 \\
\hline \multicolumn{2}{|c|}{ 04RR153DBear Lake PP } & 55.9 & 041RR139D & \multicolumn{2}{|c|}{ DHerendeenPP } & $55.86117-16$ & 04RR14 ${ }^{2}$ & Shelikof & WOGC & 57.70 \\
\hline \multicolumn{2}{|c|}{ 04RR163CBear Lake PP } & $1-159.87989$ & 04RR139A ${ }^{1}$ & \multicolumn{2}{|c|}{$A^{1}$ HerendeenHSG } & $55.86117-160$ & 04RR15B & Shelikof & PP & 57.70040 \\
\hline 04RR8B & Bear Lake PP & 57.5767 & $04 R$ & \multicolumn{2}{|c|}{ HerendeenPP } & 55.8611 & 15D & Shelikof & PP & 57.700 \\
\hline 04RR8D & Bear Lake PP & 57. & $04 R$ & \multicolumn{2}{|c|}{ HerendeenPP } & 55 & 04 & likof & $\mathrm{PP}$ & 5723 \\
\hline 04RR8E & Bear Lake PP & 220 & $56 C$ & \multicolumn{2}{|c|}{ HerendeenTOC } & & 16B & likof & $\mathrm{PP}$ & 6636 \\
\hline CP1-11 & Bear Lake PP & 55 & 156D & \multicolumn{2}{|c|}{ HerendeenPP } & & 18B & Shelikof & PP & 3261 \\
\hline CP1-138 & Bear Lake PP & 55.78 & $\mathrm{HS} 1-12$ & \multicolumn{2}{|c|}{ HerendeenPP } & 55. & 04RR18D & Shelikof & PP & 3261 \\
\hline CP1-178 & Bear Lake PP & $55.78596-160.87172$ & HS1-16 & \multicolumn{2}{|c|}{ HerendeenPP } & 55.8 & 04RR1D & Shelikof & $\mathrm{PP}$ & $57.7 \varepsilon$ \\
\hline CP1-26 & Bear Lake PP & $6-160.87172$ & HS1-2.5 & \multicolumn{2}{|c|}{ HerendeenPP } & 55.8 & 04RR28B & Shelikof & PP & 8372 \\
\hline CP1-38 & Bear Lake PP & 55.7 & HS1 & \multicolumn{2}{|c|}{ HerendeenPP } & 55. & 04RR29A & Shelikof & $\mathrm{PP}$ & 57.7 \\
\hline CP1-92 & Bear Lake PP & $55.78596-160.87172$ & HS1-8.5 & \multicolumn{2}{|c|}{ HerendeenPP } & $55.85998-160.49368$ & 04RR30B & Shelikof & PP & $57.71912-155.68790$ \\
\hline LH1-120 & Bear Lake PP & $55.87441-160.34538$ & 04RR12B & Naknek & PP & $57.53561-156.75188$ & O4RR30D & Shelikof & $\mathrm{PP}$ & $2-155.68790$ \\
\hline LH1-164 & Bear Lake PP & $55.87441-160.34538$ & 04RR157B & Naknek & PP & $55.75607-160.69526$ & 04DS04B & Kialagvik & $\mathrm{PP}$ & $51-155.40000$ \\
\hline LH1-182 & Bear Lake PP & 55.8 & $04 \mathrm{R}$ & Naknek & PP & 57.4 & S09C & Kialagvik & PP & 57. \\
\hline LH1-2 & Bear Lake PP & $55.87441-16$ & 04RR20B & Naknek & PP & $57.41584-156.50539$ & 04RR11A & Kialagvik & TOC & $57.30629-156.42686$ \\
\hline LH1-66.5 & Bear Lake PP & $55.87441-160.34538$ & 04RR20C & Naknek & $I G$ & $57.41584-156.50539$ & 04RR11B & Kialagvik & TOC & $57.30629-156.42686$ \\
\hline LH1-98 & Bear Lake PP & $55.87441-160.34538$ & 04RR22B & Naknek & PP & $57.76649-155.63527$ & 04RR11D & Kialagvik & PP & $57.30629-156.42686$ \\
\hline SD1-10 & Bear Lake PP & 55.8 & 04RR23A & Naknek & PP & $57.76330-1$ & 04RR3B & Kialagvik & $\mathrm{PP}$ & 0.39858 \\
\hline SD1-41 & Bear Lake PP & $55.81693-160.31337$ & 04RR26B & Naknek & PP & $57.74717-155.68050$ & 04RR9B & Kialagvik & PP & $57.39350-156.37367$ \\
\hline SD1-56 & Bear Lake PP & $55.81693-160.31337$ & 04RR2C & Naknek & PP & $59.79255-155.60508$ & 04TJR08 & Kialagvik & PP & $57.30766-156.43205$ \\
\hline SD1-64 & Bear Lake PP & $55.81693-160.31337$ & O4RR2D & Naknek & PP & 59.79255-155.60508 & 04TJR08 & Kialagvik & TOC & $57.30766-156.43205$ \\
\hline \multirow[t]{2}{*}{ SD1-86 } & Bear Lake PP & $55.81693-160.31337$ & 04RR6B & Naknek & PP & $57.65384-156.27242$ & 04DS13A & Kamishak & TOC & $57.71427-155.36745$ \\
\hline & & & 04RR6D & Naknek & $\mathrm{PP}$ & $57.65384-156.27242$ & 04DS14 & Kamishak & TOC & $57.71432-155.37116$ \\
\hline
\end{tabular}

Table 1. List of samples and analyses performed. ${ }^{1}$ Gas Seep; ${ }^{2}$ Oil Seep; TOC-total organic carbon; PP-porosity and permeability; WOGC-whole oil geochemistry; HSG-headspace gas analysis. 


\begin{tabular}{|lll|}
\hline Analysis & Result & Units/Formula \\
C1 & 677881 & parts per million by mole \\
C2 & 5842 & parts per million by mole \\
C3 & 173 & parts per million by mole \\
iC4 & 6 & parts per million by mole \\
C4 & 3 & parts per million by mole \\
iC5 & & parts per million by mole \\
C5 & 0 & parts per million by mole \\
C6+ & 7 & parts per million by mole \\
Gas & 99.1 & C1 \\
Composition & 0.9 & C2-C4 \\
Percent & 0.0 & C5-C6+ \\
Wet Gas \% & 0.9 & C2-C4 / C1-C4 \\
Gas Maturity & 2.1 & i-C4 / n-C4 \\
\hline
\end{tabular}

Table 2. Headspace Gas analysis performed on sample 04RR139A (gas seep in Herendeen Formations; 139 on Figure 3) by Baseline DGSI.

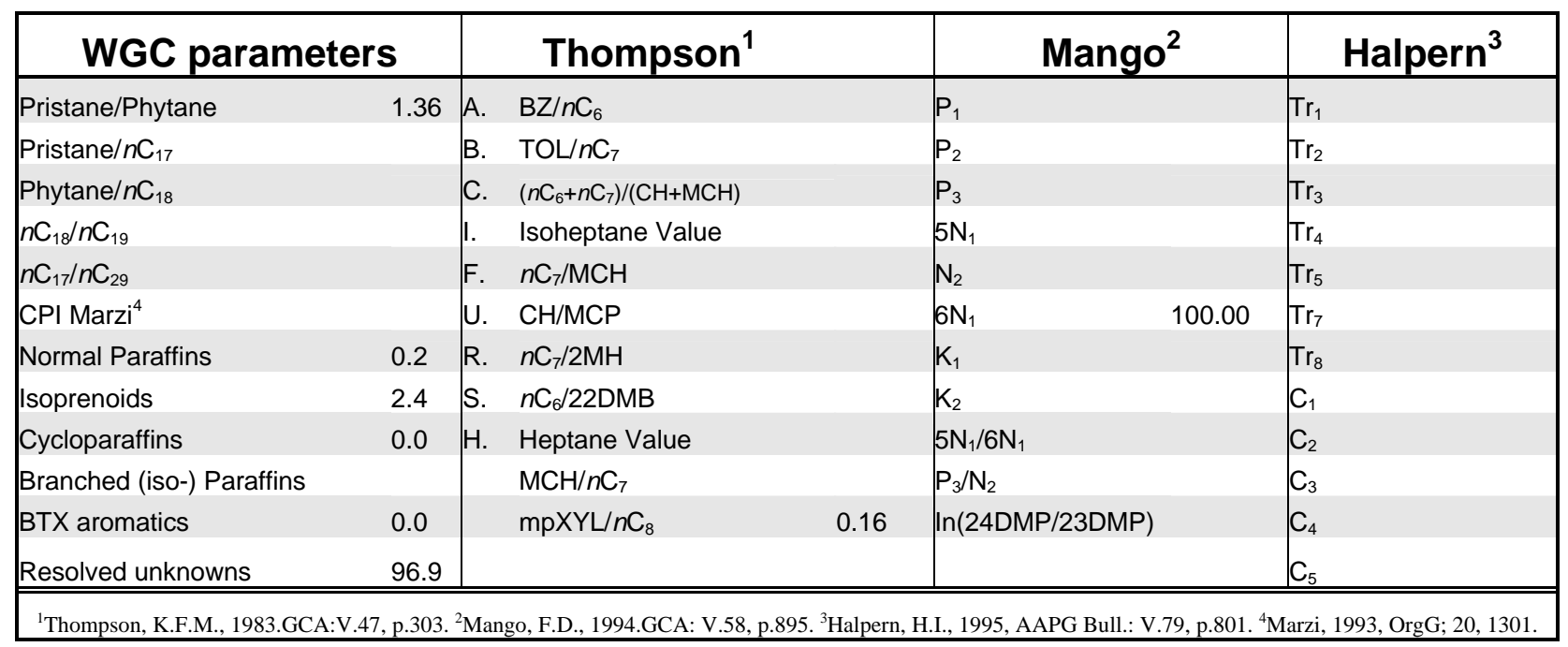

Table 3a. Parameters used in whole oil geochemical analysis.

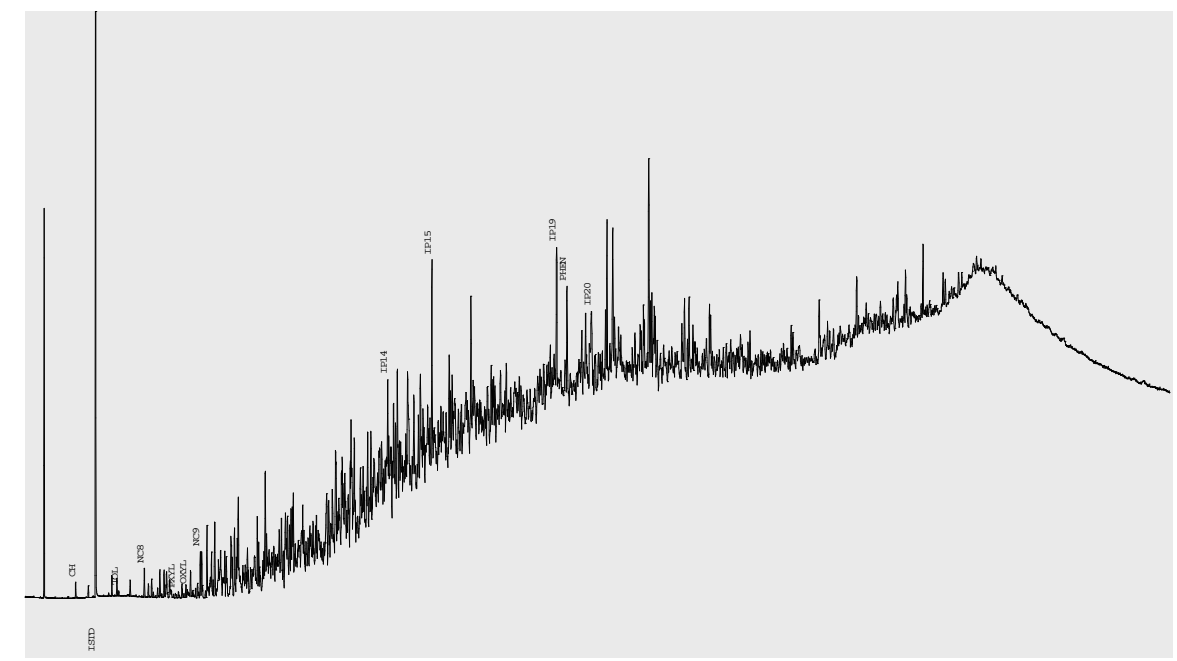

Table 3b. Whole oil geochemical traces. 


\begin{tabular}{|c|c|c|c|c|c|c|c|c|c|c|c|c|c|}
\hline $\begin{array}{l}\text { Peak } \\
\text { Label }\end{array}$ & $\begin{array}{l}\text { Compound } \\
\text { Name } \\
\end{array}$ & $\begin{array}{l}\text { Ret. } \\
\text { Time } \\
\end{array}$ & Area & Height & $\begin{array}{l}\text { ppt } \\
\text { (Area) } \\
\end{array}$ & $\begin{array}{l}\text { ppt } \\
\text { (Height) }\end{array}$ & $\begin{array}{l}\text { Peak } \\
\text { Label }\end{array}$ & $\begin{array}{l}\text { Compound } \\
\text { Name } \\
\end{array}$ & $\begin{array}{l}\text { Ret. } \\
\text { Time }\end{array}$ & Area & Height & $\begin{array}{l}\mathrm{ppt} \\
\text { (Area) } \\
\end{array}$ & $\begin{array}{l}\text { ppt } \\
\text { (Height) }\end{array}$ \\
\hline IC4 & Iso-alkane C4 & & & & & & ISTD & Internal Standard & 10.296 & 87676 & 39522 & 3.44 & 3.44 \\
\hline NC4 & Normal Alkane C4 & & & & & & $\mathrm{MCH}$ & Methylcyclohexane & & & & & \\
\hline IC5 & Iso-alkane C5 & & & & & & 113TMCP & 1,1,3,-Trimethylcyclopentane & & & & & \\
\hline NC5 & Normal Alkane C5 & & & & & & ECP & Ethylcyclopentane & & & & & \\
\hline 22DMB & 2,2-Dimethylbutane & & & & & & 124TMCP & 1,2,4-Trimethylcyclopentane & & & & & \\
\hline $\mathrm{CP}$ & Cyclopentane & & & & & & 123TMCP & 1,2,3-Trimethylcyclopentane & & & & & \\
\hline 23DMB & 2,3-Dimethylbutane & & & & & & TOL & Toluene & 12.705 & 223 & 72 & 0.01 & 0.01 \\
\hline $2 \mathrm{MP}$ & 2-Methylpentane & & & & & & NC8 & Normal Alkane C8 & 15.345 & 1066 & 396 & 0.04 & 0.03 \\
\hline $3 \mathrm{MP}$ & 3-Methylpentane & & & & & & IP9 & Isoprenoid C9 & & & & & \\
\hline NC6 & Normal Alkane C6 & & & & & & MXYL & m-Xylene & & & & & \\
\hline 22DMP & 2,2-Dimethylpentane & & & & & & PXYL & p-Xylene & 18.563 & 172 & 61 & 0.01 & 0.01 \\
\hline MCP & Methylcyclopentane & & & & & & OXYL & o-Xylene & 19.794 & 390 & 115 & 0.02 & 0.01 \\
\hline 24DMP & 2,4-Dimethylpentane & & & & & & NC9 & Normal Alkane C9 & 21.128 & 1965 & 634 & 0.08 & 0.06 \\
\hline 223TMB & 2,2,3-Trimethylbutane & & & & & & IP10 & Isoprenoid C10 & & & & & \\
\hline BZ & Benzene & & & & & & $\mid \mathrm{P} 11$ & Isoprenoid C11 & & & & & \\
\hline 33DMP & 3,3-Dimethylpentane & & & & & & IP13 & Isoprenoid C13 & & & & & \\
\hline $\mathrm{CH}$ & Cyclohexane & 8.242 & 465 & 220 & 0.02 & 0.02 & IP14 & Isoprenoid C14 & 40.529 & 8416 & 2029 & 0.33 & 0.18 \\
\hline $2 \mathrm{MH}$ & 2-Methylhexane & & & & & & IP15 & Isoprenoid C15 & 45.103 & 12834 & 3044 & 0.5 & 0.27 \\
\hline 23DMP & 2,3-Dimethylpentane & & & & & & IP16 & Isoprenoid C16 & & & & & \\
\hline 11DMCP & 1,1-Dimethylcyclopentane & & & & & & IP18 & Isoprenoid C18 & & & & & \\
\hline $3 \mathrm{MH}$ & 3-Methylhexane & & & & & & IP19 & Isoprenoid C19 (Pristane) & 58 & 13266 & 2288 & 0.52 & 0.2 \\
\hline 1C3DMCP & 1-cis-3-Dimethylcyclopentane & & & & & & PHEN & Phenanthrene & 59.081 & 8044 & 1691 & 0.32 & 0.15 \\
\hline 1T3DMCP & 1-trans-3-Dimethylcyclopentane & & & & & & IP20 & Isoprenoid C20 (Phytane) & 61.616 & 9720 & 1162 & 0.38 & 0.1 \\
\hline 3EP & 3-Ethylpentane & & & & & & $\mathrm{C} 25 \mathrm{HBI}$ & $\begin{array}{l}\text { Highly Branch Isoprenoid } \\
\text { C25 }\end{array}$ & & & & & \\
\hline 1T2DMCP & 1-trans-2-Dimethylcyclopentane & & & & & & $\begin{array}{l}\mathrm{NC} 22 \text { to } \\
\mathrm{NC} 41\end{array}$ & $\begin{array}{l}\text { Normal Alkane C10 to } \\
\text { Normal Alkane C41 }\end{array}$ & No & Results & & & \\
\hline NC7 & Normal Alkane C7 & & & & & & & & & & & & \\
\hline
\end{tabular}

Table 3c. Whole oil geochemical analysis results. 


\begin{tabular}{|c|c|}
\hline Parameter & Formula \\
\hline \multicolumn{2}{|l|}{ WGC Parameters } \\
\hline Pristane/Phytane & IP19/IP20 \\
\hline Pristane/nC17 & IP19/NC17 \\
\hline Phytane/nC18 & IP20/NC18 \\
\hline nC18/nC19 & NC18/NC19 \\
\hline nC17/nC29 & NC17/NC29 \\
\hline CPI Marzi ${ }^{4}$ & $((\mathrm{NC} 23+\mathrm{NC} 25+\mathrm{NC} 27)+(\mathrm{NC} 25+\mathrm{NC} 27+\mathrm{NC} 29)) /(2 *(\mathrm{NC} 24+\mathrm{NC} 26+\mathrm{NC} 28))$ \\
\hline Normal Paraffins & $\begin{array}{l}100 *(\mathrm{NC} 4+\mathrm{NC} 5+\mathrm{NC} 6+\mathrm{NC} 7+\mathrm{NC} 8+\mathrm{NC} 9+\mathrm{NC} 10+\mathrm{NC} 11+\mathrm{NC} 12+\mathrm{NC} 13+\mathrm{NC} 14+\mathrm{NC} 15+\mathrm{NC} 16+\mathrm{NC} 17+\mathrm{NC} 18+\mathrm{NC} \\
19+\mathrm{NC} 20+\mathrm{NC} 21+\mathrm{NC} 22+\mathrm{NC} 23+\mathrm{NC} 24+\mathrm{NC} 25+\mathrm{NC} 26+\mathrm{NC} 27+\mathrm{NC} 28+\mathrm{NC} 29+\mathrm{NC} 30+\mathrm{NC} 31+\mathrm{NC} 32+\mathrm{NC} 3+\mathrm{NC} 3 \\
4+\mathrm{NC} 35+\mathrm{NC} 36+\mathrm{NC} 37+\mathrm{NC} 38+\mathrm{NC} 39+\mathrm{NC} 40+\mathrm{NC} 41) / \mathrm{TOTAL} \text { RESOLVED.F(0.0) }\end{array}$ \\
\hline Isoprenoids & 100*(IP9+IP10+IP11+IP13+IP14+IP15+IP16+IP18+IP19+IP20+CH25HBI)/TOTAL_RESOLVED \\
\hline Cycloparaffins & $\begin{array}{l}100^{*}(\mathrm{CP}+\mathrm{MCP}+\mathrm{CH}+11 \mathrm{DMCP}+1 \mathrm{~T} 3 \mathrm{DMCP}+1 \mathrm{~T} 2 \mathrm{DMCP}+\mathrm{MCH}+113 \mathrm{TMCP}+\mathrm{ECP}+124 \mathrm{TMCP}+123 \mathrm{TMCP}+1 \mathrm{C} 3 \mathrm{D} \\
\mathrm{MCP}+1 \mathrm{C} 2 \mathrm{DMCP}) / \mathrm{TOTAL} \text { RESOLVED }\end{array}$ \\
\hline $\begin{array}{l}\text { Branched (iso-) } \\
\text { Paraffins }\end{array}$ & $\begin{array}{l}\text { 100*(IC4+IC5+22DMB+23DMB+2MP+3MP+22DMP+24DMP+223TMB+33DMP+2MH+23DMP+3MH+3EP }) \\
\text { /TOTAL_RESOLVED }\end{array}$ \\
\hline BTX aromatics & $100^{*}(\mathrm{BZ}+\mathrm{TOL}+\mathrm{XYL}) / \mathrm{TOTAL} R \mathrm{RESOLVED}$ \\
\hline \multicolumn{2}{|l|}{ Thompson ${ }^{1}$} \\
\hline $\mathrm{BZ} / \mathrm{nC6}$ & BZ/NC6 \\
\hline TOL/nC7 & TOL/NC7 \\
\hline$(\mathrm{nC} 6+\mathrm{nC} 7) /(\mathrm{CH}+\mathrm{MCH})$ & $(\mathrm{NC} 6+\mathrm{NC} 7) /(\mathrm{CH}+\mathrm{MCH})$ \\
\hline Isoheptane Value & $(2 \mathrm{MH}+3 \mathrm{MH}) /(1 \mathrm{C} 3 \mathrm{DMCP}+1 \mathrm{~T} 3 \mathrm{DMCP}+1 \mathrm{~T} 2 \mathrm{DMCP})$ \\
\hline $\mathrm{nC} 7 / \mathrm{MCH}$ & $\mathrm{NC7} / \mathrm{MCH}$ \\
\hline $\mathrm{CH} / \mathrm{MCP}$ & $\mathrm{CH} / \mathrm{MCP}$ \\
\hline $\mathrm{nC} 7 / 2 \mathrm{MH}$ & $\mathrm{NC7/2MH}$ \\
\hline nC6/22DMB & NC6/22DMB \\
\hline Heptane Value & $\mathrm{NC} 7^{*} 100 /(\mathrm{CH}+2 \mathrm{MH}+23 \mathrm{DMP}+11 \mathrm{DMCP}+3 \mathrm{MH}+1 \mathrm{C} 3 \mathrm{DMCP}+1 \mathrm{~T} 3 \mathrm{DMCP}+1 \mathrm{~T} 2 \mathrm{DMCP}+\mathrm{MCH}+\mathrm{NC} 7)$ \\
\hline $\mathrm{MCH} / \mathrm{nC} 7$ & $\mathrm{MCH} / \mathrm{NC7}$ \\
\hline $\mathrm{mpXYL/nC8}$ & $\mathrm{XYL} / \mathrm{NC8}$ \\
\hline \multicolumn{2}{|l|}{ Mango $^{2}$} \\
\hline $\mathrm{P} 1$ & $\begin{array}{l}(100 * \mathrm{NC} 7) /(22 \mathrm{DMP}+24 \mathrm{DMP}+223 \mathrm{TMB}+33 \mathrm{DMP}+2 \mathrm{MH}+23 \mathrm{DMP}+11 \mathrm{DMCP}+3 \mathrm{MH}+1 \mathrm{C} 3 \mathrm{DMCP}+1 \mathrm{~T} 3 \mathrm{DMCP}+3 \mathrm{E} \\
\mathrm{P}+1 \mathrm{~T} 2 \mathrm{DMCP}+\mathrm{NC}+\mathrm{MCH}+\mathrm{ECP}+\mathrm{TOL})\end{array}$ \\
\hline $\mathrm{P} 2$ & $\begin{array}{l}100 *(2 M H+3 M H) /(22 D M P+24 D M P+223 T M B+33 D M P+2 M H+23 D M P+11 D M C P+3 M H+1 C 3 D M C P+1 T 3 D M C \\
P+3 E P+1 T 2 D M C P+N C 7+M C H+E C P+T O L)\end{array}$ \\
\hline P3 & $\begin{array}{l}100^{*}(3 \mathrm{EP}+33 \mathrm{DMP}+23 \mathrm{DMP}+24 \mathrm{DMP}+22 \mathrm{DMP}+223 \mathrm{TMB}) /(22 \mathrm{DMP}+24 \mathrm{DMP}+223 \mathrm{TMB}+33 \mathrm{DMP}+2 \mathrm{MH}+23 \mathrm{DMP} \\
+11 \mathrm{DMCP}+3 \mathrm{MH}+1 \mathrm{C} 3 \mathrm{DMCP}+1 \mathrm{~T} 3 \mathrm{DMCP}+3 \mathrm{EP}+1 \mathrm{~T} 2 \mathrm{DMCP}+\mathrm{NC}+\mathrm{MCH}+\mathrm{ECP}+\mathrm{TOL})\end{array}$ \\
\hline $5 \mathrm{~N} 1$ & $\begin{array}{l}100^{*}(\mathrm{ECP}+1 \mathrm{~T} 2 \mathrm{DMCP}) /(22 \mathrm{DMP}+24 \mathrm{DMP}+223 \mathrm{TMB}+33 \mathrm{DMP}+2 \mathrm{MH}+23 \mathrm{DMP}+11 \mathrm{DMCP}+3 \mathrm{MH}+1 \mathrm{C} 3 \mathrm{DMCP}+1 \mathrm{~T} \\
3 \mathrm{DMCP}+3 \mathrm{EP}+1 \mathrm{~T} 2 \mathrm{DMCP}+\mathrm{NC}+\mathrm{MCH}+\mathrm{ECP}+\mathrm{TOL})\end{array}$ \\
\hline N2 & $\begin{array}{l}100^{*}(11 \mathrm{DMCP}+1 \mathrm{C} 3 \mathrm{DMCP}+1 \mathrm{~T} 3 \mathrm{DMCP}) /(22 \mathrm{DMP}+24 \mathrm{DMP}+223 \mathrm{TMB}+33 \mathrm{DMP}+2 \mathrm{MH}+23 \mathrm{DMP}+11 \mathrm{DMCP}+3 \mathrm{MH} \\
+1 \mathrm{C} 3 \mathrm{DMCP}+1 \mathrm{~T} 3 \mathrm{DMCP}+3 \mathrm{EP}+1 \mathrm{~T} 2 \mathrm{DMCP}+\mathrm{NC} 7+\mathrm{MCH}+\mathrm{ECP}+\mathrm{TOL})\end{array}$ \\
\hline $6 \mathrm{~N} 1$ & $\begin{array}{l}100 *(\mathrm{MCH}+\mathrm{TOL}) /(22 \mathrm{DMP}+24 \mathrm{DMP}+223 \mathrm{TMB}+33 \mathrm{DMP}+2 \mathrm{MH}+23 \mathrm{DMP}+11 \mathrm{DMCP}+3 \mathrm{MH}+1 \mathrm{C} 3 \mathrm{DMCP}+1 \mathrm{~T} 3 \mathrm{DMC} \\
\mathrm{P}+3 \mathrm{EP}+1 \mathrm{~T} 2 \mathrm{DMCP}+\mathrm{NC} 7+\mathrm{MCH}+\mathrm{ECP}+\mathrm{TOL})\end{array}$ \\
\hline K1 & $(2 \mathrm{MH}+23 \mathrm{DMP}) /(3 \mathrm{MH}+24 \mathrm{DMP})$ \\
\hline K2 & $(2 \mathrm{MH}+3 \mathrm{MH}) /(2 \mathrm{MH}+3 \mathrm{MH}+11 \mathrm{DMCP}+1 \mathrm{C} 3 \mathrm{DMCP}+1 \mathrm{~T} 3 \mathrm{DMCP})$ \\
\hline $5 \mathrm{~N} 1 / 6 \mathrm{~N} 1$ & $(\mathrm{ECP}+1 \mathrm{~T} 2 \mathrm{DMCP}) /(\mathrm{MCH}+\mathrm{TOL})$ \\
\hline $\mathrm{P} 3 / \mathrm{N} 2$ & $(3 E P+33 D M P+23 D M P+24 D M P+22 D M P+223 T M B) /(11 D M C P+1 C 3 D M C P+1 T 3 D M C P)$ \\
\hline $\ln (24 D M P / 23 D M P)$ & $\ln (24 D M P / 23 D M P)$ \\
\hline \multicolumn{2}{|l|}{ Halpern $^{3}$} \\
\hline $\operatorname{Tr} 1$ & TOL/11DMCP \\
\hline Tr2 & NC7/11DMCP \\
\hline Tr3 & 3MH/11DMCP \\
\hline Tr4 & $2 \mathrm{MH} / 11 \mathrm{DMCP}$ \\
\hline $\operatorname{Tr} 5$ & $(2 \mathrm{MH}+3 \mathrm{MH}) / 11 \mathrm{DMCP}$ \\
\hline $\operatorname{Tr} 7$ & 1T3DMCP/11DMCP \\
\hline $\operatorname{Tr} 8$ & $(2 \mathrm{MH}+3 \mathrm{MH}) /(22 \mathrm{DMP}+23 \mathrm{DMP}+24 \mathrm{DMP}+33 \mathrm{DMP}+3 \mathrm{EP})$ \\
\hline C1 & $22 \mathrm{DMP} /(22 \mathrm{DMP}+23 \mathrm{DMP}+24 \mathrm{DMP}+33 \mathrm{DMP}+3 E P)$ \\
\hline $\mathrm{C} 2$ & 23DMP/(22DMP+23DMP+24DMP+33DMP+3EP) \\
\hline C3 & 24DMP/(22DMP+23DMP+24DMP+33DMP+3EP) \\
\hline C4 & 33DMP/(22DMP+23DMP+24DMP+33DMP+3EP) \\
\hline $\mathrm{C5}$ & $3 E P /(22 D M P+23 D M P+24 D M P+33 D M P+3 E P)$ \\
\hline
\end{tabular}

Table 3d. Whole oil geochemical formulas. 


\begin{tabular}{|c|c|c|c|c|c|c|c|c|c|c|c|c|}
\hline Sample & Formation & $\begin{array}{l}\text { Sample } \\
\text { Type }\end{array}$ & $\begin{array}{l}\text { TOC } \\
\text { Wt. \% }\end{array}$ & $\begin{array}{l}\mathrm{s} 1 \\
\mathrm{mg} / \mathrm{g}\end{array}$ & $\begin{array}{l}\mathrm{S} 2 \\
\mathrm{mg} / \mathrm{g}\end{array}$ & $\begin{array}{l}\text { S3 } \\
\mathrm{mg} / \mathrm{g}\end{array}$ & Tmax & HI & OI & $\begin{array}{l}\text { S1/ } \\
\text { TOC }\end{array}$ & PI & Verified \\
\hline 04RR140B & Bear Lake & Outcrop & 0.21 & 0.02 & 0.07 & 0.34 & 464 & 33 & 162 & 9 & 0.22 & \\
\hline 04RR140E & Bear Lake & Outcrop & 0.08 & 0.09 & 0.07 & 0.44 & 520 & 88 & 550 & 111 & 0.56 & \\
\hline 04RR138D & Stepovak & Outcrop & 9.54 & 0.04 & 0.41 & 0.41 & 548 & 4 & 4 & 0 & 0.09 & TOC \\
\hline 04RR142A & Chignik & Outcrop & 0.02 & 0.02 & 0.10 & 0.26 & 526 & 500 & 1300 & 98 & 0.16 & \\
\hline 04-TJR-4G & Naknek* & Outcrop & 2.19 & 0.03 & 8.49 & 0.51 & 420 & 389 & 23 & 1 & 0.00 & TOC RE \\
\hline 04-DS-10A & Shelikof & Outcrop & 0.20 & 0.00 & 0.10 & 0.26 & 497 & 50 & 130 & 0 & 0.00 & \\
\hline 04-DS-10B & Shelikof & Outcrop & 0.18 & 0.01 & 0.10 & 0.13 & 544 & 56 & 72 & 5 & 0.09 & \\
\hline 04-DS-11 & Shelikof & Outcrop & 0.36 & 0.01 & 0.25 & 0.19 & 428 & 69 & 53 & 3 & 0.04 & \\
\hline 04-RR-13C & Shelikof & Outcrop & 0.23 & 0.02 & 0.17 & 0.15 & 545 & 74 & 65 & 9 & 0.10 & \\
\hline 04-RR-11A & Kialagvik & Outcrop & 0.81 & 0.30 & 0.58 & 0.32 & 444 & 72 & 40 & 37 & 0.34 & \\
\hline 04-RR-11B & Kialagvik & Outcrop & 0.57 & 0.09 & 0.33 & 0.28 & 465 & 58 & 49 & 16 & 0.21 & \\
\hline 04-TJR-08 & Kialagvik & Outcrop & 0.54 & 0.08 & 0.33 & 0.13 & 461 & 61 & 24 & 15 & 0.19 & \\
\hline 04-DS-13A & Kamishak & Outcrop & 2.38 & 1.28 & 14.24 & 0.50 & 429 & 598 & 21 & 54 & 0.08 & TOC RE \\
\hline 04-DS-14 & Kamishak & Outcrop & 1.12 & 0.33 & 5.31 & 0.25 & 425 & 474 & 22 & 30 & 0.06 & TOC RE \\
\hline
\end{tabular}

Table 4. Total organic carbon, pyrolysis analysis of 15 outcrop samples performed by Baseline DGSI. 




Figure 6. Kerogen type determination of total organic carbon outcrop samples. Types I and II will generate oil, type III gas, and type IV little or no hydrocarbons. *Snug Harbor Member. 


\begin{tabular}{|c|c|c|c|c|c|c|c|c|c|c|c|c|c|}
\hline \multicolumn{3}{|c|}{ Porosity } & \multicolumn{2}{|c|}{ Permeability (millidarcies) } & \multicolumn{2}{|r|}{ Grain Density } & \multicolumn{3}{|c|}{ Porosity } & \multicolumn{2}{|c|}{ Permeability (millidarcies) } & \multicolumn{2}{|r|}{ Grain Density } \\
\hline Sample ID & $\%$ & Formation & Klinkenberg & Kair & Probe & $\mathrm{g} / \mathrm{cm}^{3}$ & Sample ID & $\%$ & Formation & Klinkenberg & Kair & Probe & $\mathbf{G} / \mathbf{c m}^{3}$ \\
\hline 04RR140C & 11.7 & Bear Lake & 0.613 & 0.898 & & 2.53 & 04RR11D & 3.9 & Kialagvik & 0.060 & 0.106 & & 2.63 \\
\hline SD1-56 & 8.3 & Bear Lake & 0.003 & 0.010 & & 2.71 & 04TJR08 & 7.4 & Kialagvik & 0.444 & 0.698 & & 2.76 \\
\hline LH1-164 & 4.4 & Bear Lake & 0.005 & 0.013 & & 2.73 & 04DS04B & 4.9 & Kialagvik & ns & ns & 0.021 & 2.70 \\
\hline CP1-138 & 7.5 & Bear Lake & 0.006 & 0.017 & & 2.71 & 04DS09C & 4.8 & Kialagvik & ns & ns & 0.053 & 2.74 \\
\hline SD1-64 & 10.6 & Bear Lake & 0.024 & 0.048 & & 2.69 & 04RR3B & 13.4 & Kialagvik & ns & ns & 0.027 & 2.70 \\
\hline CP1-38 & 6.2 & Bear Lake & 0.027 & 0.053 & & 2.66 & SR2-6 & 35.4 & Milky River & 62.237 & 73.113 & & 2.75 \\
\hline LH1-120 & 8.3 & Bear Lake & 0.032 & 0.061 & & 2.67 & SR1-3.5 & 32.7 & Milky River & 222.139 & 245.875 & & 2.68 \\
\hline CP1-92 & 9.6 & Bear Lake & 0.041 & 0.076 & & 2.69 & SR2-89.5 & 35.3 & Milky River & 3425.436 & 3553.977 & & 2.70 \\
\hline LH1-66.5 & 6.5 & Bear Lake & 0.055 & 0.098 & & 2.67 & 04RR154B & 42.0 & Milky River & ns & ns & & 2.81 \\
\hline 04RR148B & 9.8 & Bear Lake & 0.063 & 0.110 & & 2.68 & 04RR158C & 35.0 & Milky River & ns & ns & 125.000 & 2.73 \\
\hline SD1-86 & 4.9 & Bear Lake & 0.184 & 0.280 & & 2.68 & |04RR158G & 35.6 & Milky River & ns & ns & 76.600 & 2.63 \\
\hline CP1-11 & 13.6 & Bear Lake & 0.190 & 0.288 & & 2.66 & 04RR6B & 2.7 & Naknek & 0.002 & 0.008 & & 2.51 \\
\hline SD1-10 & 10.8 & Bear Lake & 0.191 & 0.310 & & 2.66 & 04RR23A & 1.5 & Naknek & 0.005 & 0.014 & & 2.68 \\
\hline 04RR152C & 11.9 & Bear Lake & 0.242 & 0.377 & & 2.72 & 04TJR09 & 5.3 & Naknek & 0.026 & 0.051 & & 2.52 \\
\hline LH1-2 & 8.9 & Bear Lake & 0.474 & 0.691 & & 2.68 & 04RR2C & 3.8 & Naknek & 0.074 & 0.126 & & 2.63 \\
\hline CP1-26 & 11.2 & Bear Lake & 0.582 & 0.870 & & 2.64 & 04RR12B & 5.0 & Naknek & 0.114 & 0.184 & & 2.61 \\
\hline 04RR8B & 36.1 & Bear Lake & $\mathrm{ns}$ & ns & 41.4 & 2.63 & 04RR2D & 4.6 & Naknek & 0.137 & 0.216 & & 2.60 \\
\hline 04RR8D & 39.6 & Bear Lake & ns & ns & 0.251 & 2.49 & 04RR7D & 4.5 & Naknek & 1.562 & 2.195 & & 2.56 \\
\hline 04RR8E & 38.4 & Bear Lake & ns & ns & 0.066 & 2.46 & 04RR19B & 6.3 & Naknek & 2.433 & 3.382 & & 2.62 \\
\hline 04RR153D & 9.2 & Bear Lake & ns & ns & 0.180 & 2.71 & 04RR26B & 4.6 & Naknek & 3.829 & 5.366 & & 2.61 \\
\hline LH1-98 & 16.9 & Bear Lake & ns & ns & 0.065 & 2.69 & O4RR20B & 13.9 & Naknek & ns & ns & 0.223 & 2.74 \\
\hline LH1-182 & 11.3 & Bear Lake & ns & ns & 0.037 & 2.62 & 04RR22B & 6.5 & Naknek & ns & ns & 48.1 & 2.56 \\
\hline SD1-41 & 10.0 & Bear Lake & ns & ns & 0.08 & 2.68 & 04RR6D & 7.2 & Naknek & ns & ns & 20.0 & 2.71 \\
\hline 04RR163C & 14.1 & Bear Lake & ns & ns & 0.448 & 2.73 & 04RR7B & 7.2 & Naknek & ns & ns & 37.7 & 2.57 \\
\hline CP1-178 & 11.2 & Bear Lake & ns & ns & 0.870 & 2.66 & 04RR157B & 8.1 & Naknek & 44.113 & 52.914 & & 2.59 \\
\hline 04RR150B & 2.6 & Chignik & 0.001 & 0.004 & & 2.71 & 04RR30B & 3.5 & Shelikof & 0.002 & 0.006 & & 2.70 \\
\hline 04RR141B & 13.8 & Chignik & 0.008 & 0.020 & & 2.69 & 04RR18B & 3.6 & Shelikof & 0.004 & 0.010 & & 2.62 \\
\hline 04RR149B & 4.6 & Chignik & 0.308 & 0.447 & & 2.71 & 04RR1D & 6.7 & Shelikof & 0.005 & 0.014 & & 2.63 \\
\hline 04RR151C & 7.1 & Chignik & 1.230 & 1.694 & & 2.66 & 04RR16B & 3.7 & Shelikof & 0.006 & 0.016 & & 2.73 \\
\hline 04RR142B & 12.4 & Chignik & $\mathrm{ns}$ & $\mathrm{ns}$ & 1.950 & 2.68 & 04RR15G & 5.3 & Shelikof & 0.008 & 0.019 & & 2.73 \\
\hline 04RR156B & 4.6 & Herendeen & 0.001 & 0.004 & & 2.70 & 04RR29A & 5.2 & Shelikof & 0.008 & 0.020 & & 2.65 \\
\hline 04RR156D & 1.1 & Herendeen & 0.002 & 0.006 & & 2.68 & 04RR28B & 7.7 & Shelikof & 0.043 & 0.079 & & 2.80 \\
\hline HS1-16 & 7.3 & Herendeen & 0.002 & 0.007 & & 2.67 & 04RR15B & 8.5 & Shelikof & 0.110 & 0.178 & & 2.73 \\
\hline HS1-8.5 & 5.1 & Herendeen & 0.007 & 0.018 & & 2.60 & 04RR15D & 6.4 & Shelikof & 0.165 & 0.254 & & 2.71 \\
\hline HS1-2.5 & 7.9 & Herendeen & 0.007 & 0.018 & & 2.70 & 04RR13B & 4.9 & Shelikof & 0.374 & 0.522 & & 2.69 \\
\hline HS1-12 & 5.3 & Herendeen & 0.009 & 0.022 & & 2.68 & 04RR18D & 8.4 & Shelikof & 0.446 & 0.689 & & 2.73 \\
\hline HS1-5.5 & 6.3 & Herendeen & 0.049 & 0.088 & & 2.73 & 04RR30D & 10.1 & Shelikof & ns & ns & 0.462 & 2.70 \\
\hline 041RR139D & 11.0 & Herendeen & 0.793 & 1.188 & & 2.68 & 04RR138 & 16.6 & Stepovak & 0.004 & 0.012 & & 2.70 \\
\hline 04RR139C & 11.6 & Herendeen & ns & $\mathrm{ns}$ & 1.080 & 2.64 & 04RR155B & 7.0 & Tolstoi & 0.002 & 0.005 & & 2.71 \\
\hline 04RR9B & 9.7 & Kialagvik & 0.001 & 0.005 & & 2.77 & 04RR168B & 4.4 & Tolstoi & 0.004 & 0.010 & & 2.75 \\
\hline & & & & & & & 04RR155B & 9.6 & Tolstoi & 0.099 & 0.163 & & 2.72 \\
\hline
\end{tabular}

Table 5. Porosity and permeability (conventional core analysis) of outcrop samples performed by Core Laboratories, Inc. Performed at 400 psig net confining stress. $n s=n o t$ suitable for analysis. 


\section{Porosity and Permeability of all Bristol Bay Samples}



Figure 7. Porosity and permeability of outcrop samples (undifferentiated); md - millidarcies. 


\section{Porosity and Permeability by Formation}

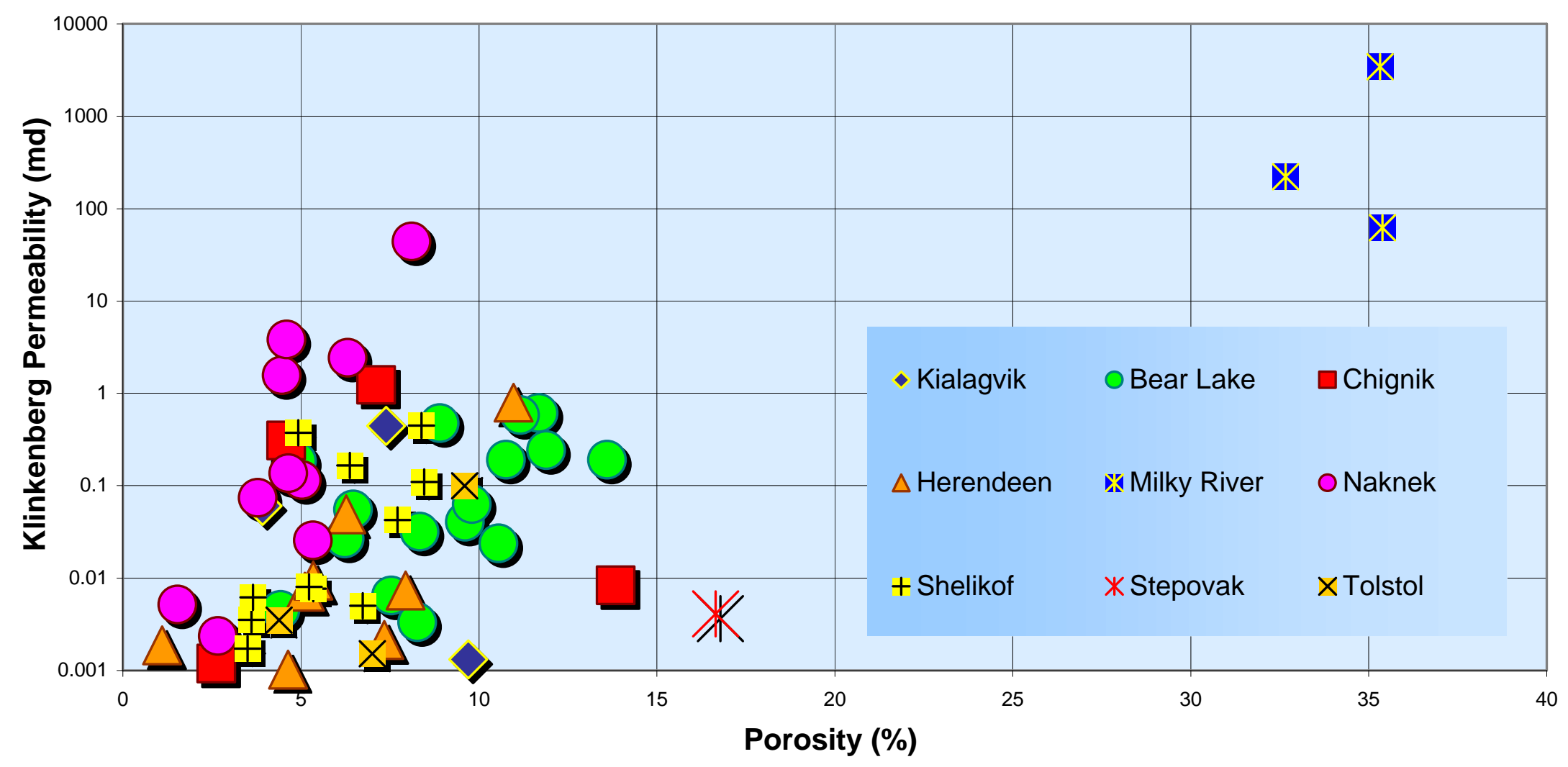

Figure 8. Porosity and permeability of outcrop samples (by formation); md - millidarcies. 


\begin{tabular}{|lcc|}
\hline Test & Result & Units \\
$\mathrm{SiO} 2$ & 52.11 & $\%$ \\
\hline $\mathrm{Al} 2 \mathrm{O} 3$ & 14.47 & $\%$ \\
$\mathrm{Fe} 2 \mathrm{O} 3$ & 11.89 & $\%$ \\
$\mathrm{CaO}$ & 5.42 & $\%$ \\
\hline $\mathrm{MgO}$ & 4.13 & $\%$ \\
$\mathrm{Na2O}$ & 1.61 & $\%$ \\
$\mathrm{~K} 2 \mathrm{O}$ & 0.84 & $\%$ \\
$\mathrm{Cr} 2 \mathrm{O} 3$ & 0.01 & $\%$ \\
\hline $\mathrm{TiO} 2$ & 1.21 & $\%$ \\
$\mathrm{MnO}$ & 0.23 & $\%$ \\
$\mathrm{P} 2 \mathrm{O} 5$ & 0.26 & $\%$ \\
$\mathrm{SrO}$ & 0.04 & $\%$ \\
\hline $\mathrm{BaO}$ & 0.06 & $\%$ \\
LOI & 7.44 & $\%$ \\
total & 99.72 & $\%$ \\
\hline FeO & 4.7 & $\%$ \\
weight sample & 327.4 & grams \\
weight heavy@2.96 & 23.007 & grams \\
weight light@2.96 & 303.3 & grams \\
weight total@2.96 & 326.3 & grams \\
heavy magnetic fraction & 18.945 & grams \\
heavy non-magnetic & & \\
fraction & 4.062 & grams \\
light magnetic fraction & 56.334 & grams \\
light non-magnetic & & \\
fraction & 246.99 & grams \\
\hline
\end{tabular}

Table 6. Inorganic geochemical analysis performed by ACME Analytical Lab, on outcrop sample 04RR20C, from the Snug Harbor Member of the Naknek Formation. 


\section{BIBLIOGRAPHY}

Albanese, M.D., Goff, K.M., 1987, Petrified wood occurrences in Alaska: Alaska Division of Geological \& Geophysical Surveys, Public Data File 87-26, 11 p.

Allaway, W.H., Jr., 1982, Sedimentology and petrography of the Shelikof Formation, Alaska Peninsula: San Jose, California, San Jose State University, unpublished M.S. thesis, 81 p.

Allaway, W.H., Jr., and Miller, J.W., 1984, Newly recognized sedimentary environments in the Shelikof Formation, in Conrad, W.L, and Elliot, R.L., eds., The United States Geological Survey in Alaska: Accomplishments during 1981: U.S. Geological Survey Circular 868, p. 34-37.

Allaway, W.H., Jr., Detterman, R.L., Miller, J.W., and Magoon, L.B., 1984, Stratigraphic clarification of the Shelikof Formation, Alaska Peninsula, in Stratigraphic notes, 1983: U.S. Geological Survey Bulletin 1537-A, p. A21-A29.

Blasko, D.P., 1976, Oil and gas seeps in Alaska: Alaska Peninsula, western Gulf of Alaska: U.S. Bureau of Mines Report of Investigations no. 8122, 78 p.

Blodgett, R.B., Weems, R.E., and Wilson, F.H., 1995, Upper Jurassic reptiles from the Naknek Formation, Alaska Peninsula; A glimpse into Alaska's own "Jurassic Park": Geological Society of America Abstracts with Programs, v. 27, no. 5, p. 6.

Burk, C.A., 1965, Geology of the Alaska Peninsula-island arc and continental margin: Geological Society of America Memoir 99, 250 p.

Capps, S.R., 1921, Preliminary report on petroleum in Alaska: U.S. Geological Survey Bulletin 719, 83 p.

Capps, S.R., 1923, The Cold Bay district: U.S. Geological Survey Bulletin 739-C, p. C77-C116.

Comer, C.D. and Martin, G.C., 1994, Petroleum potential of Mesozoic strata of the Cook Inlet outer Continental shelf planning area, Alaska: Austin, Texas, Third Symposium on Studies Related to Continental Margins-A Summary of Year-Five and Year-Six Activities, p. 172-180.

Conwell, C.N., and Triplehorn, D.M., 1978, Herendeen Bay-Chignik coals, southern Alaska Peninsula: Alaska Division of Geological \& Geophysical Surveys, Special Report 8, 15 p., 2 sheets.

Detterman, R.L., 1990, Stratigraphic correlations and interpretation of exploratory wells, Alaska Peninsula: United States Geological Survey, Open-File Report 90-279, 51 p, 2 plates.

Detterman, R.L., Case, J.E., Wilson, F.H., and Yount, M.E., 1987, Geologic map of the Ugashik, Bristol Bay, and part of Karluk quadrangles, Alaska: U.S. Geological Survey Miscellaneous Investigations Series Map I-1685, scale 1:250,000.

Detterman, R.L., Case, J.E., Wilson, F.H., Yount, M.E., and Allaway, W.H., Jr., 1983, Generalized geologic map of the Ugashik, Bristol Bay, and part of Karluk quadrangles, Alaska: U.S. Geological Survey Miscellaneous Field Studies Map MF-1539-A, 1 sheet, scale 1:250,000.

Detterman, R.L., Miller, J.W., and Case, J.E., 1985, Megafossil locality map, checklists, and pre-Quaternary stratigraphic sections of Ugashik, Bristol Bay, and part of Karluk quadrangles, Alaska: U.S. Geological Survey Miscellaneous Field Studies Map MF-1539-B, 3 sheets, scale 1:250,000.

Detterman, R.L., Miller, J.W., Case, J.E., Wilson, F.H., and Yount, M.E., 1996, Stratigraphic framework of the Alaska Peninsula: U.S. Geological Survey Bulletin 1969-A, 74 p.

DGGS Staff, 1986, Summary of existing data and potential for commercial hydrocarbon accumulations, Bristol Bay, Alaska: Alaska Division of Geological \& Geophysical Surveys, Public Data File 86-13, 20 p.

Eakins, G.R., 1970, A petrified forest on Unga Island, Alaska: Alaska Division of Mines and Geology, Special Report 3, 19 p.

Franks, S.G. and Hite, D.M., 1980, Controls on zeolite cementation in Upper Jurassic sandstones, lower Cook Inlet, Alaska: Bulletin of the American Association of Petroleum Geologists v. 64, no. 5, p. 708-709.

Imlay, R.W., and Detterman, R.L., 1977, Some Lower and Middle Jurassic beds in Puale Bay-Alinchak Bay area, Alaska Peninsula: Bulletin of the American Association of Petroleum Geologists, v. 61, p. 607-611.

Jones, D.L., Silberling, N.J., and Hillhouse, John, 1977, Wrangellia-A displaced terrane in northwestern North America: Canadian Journal of Earth Science, v. 14, p. 2565-2577.

Kellum, L.B., 1945, Jurassic stratigraphy of Alaska and petroleum exploration in northwest America: New York, Acad. Sci. Trans., ser. 2, v. 7, no. 8, p. 201-209.

Kellum, L.B., Daviess, S.N., and Swinney, C.M., 1945, Geology and oil possibilities of the southwestern part of the Wide Bay anticline, Alaska: U.S. Geological Survey Preliminary Strategic Map, 17 p., 9 pls.

Kelly, J.S., and Denman, J.M., 1972, Geological literature on the Alaska Peninsula and adjacent area: Alaska Division of Geological \& Geophysical Surveys, Special Report 20, 64 p. 
Lankford, S.M. and Magoon, L.B., 1977, Petrography of the Upper Jurassic through Oligocene sandstones in the Cape Douglas-Kamishak Hills area, lower Cook Inlet, in Johnson, K.M., ed., The United States Geological Survey in Alaska: Accomplishments during 1977, p. B60-B62.

Lyle, W.M., Dobey, P.L., 1974, Geologic evaluation of the Herendeen Bay area, Alaska Peninsula: Alaska Division of Geological \& Geophysical Surveys, Alaska Open File Report 48, 22 p., 3 sheets.

Lyle, W.M., Morehouse, J.A., Palmer, I.F., Jr., Bolm, J.G., 1979, Tertiary formations and associated Mesozoic rocks in the Alaska Peninsula area, Alaska, and their petroleum-reservoir and source-rock potential: Alaska Division of Geological \& Geophysical Surveys, Geologic Report 62, 69 p., 19 sheets.

Magoon, L.B. and Anders, D.E., 1989, Oil-to-source rock correlation using carbon-isotopic data and biological marker compounds, Cook Inlet-Alaska Peninsula, in Moldowan, J.M., Albrecht, P., and Philip, R.P., eds., Biological Markers in Sediments and Petroleum: Englewood Cliffs, Prentice Hall, p. 241-273.

Magoon, L.B., Adkison, W.L., Chmelik, F.B., Dolton, G.L., Fisher, M.A., Hampton, M.A., Sable, E.G., and Smith, R.A., 1976, Hydrocarbon potential, geologic hazards, and infrastructure for exploration and development of the lower Cook Inlet, Alaska: United States Geological Survey Open-File Report 76-449, 132 p., 1 sheet.

Martin, G.C., 1926, The Mesozoic stratigraphy of Alaska: U.S. Geological Survey Bulletin 776, 493 p.

Martin, G.C., and Katz, F.J., 1912, A geologic reconnaissance of the Iliamna region, Alaska: U.S. Geological Survey Bulletin 485, $138 \mathrm{p}$.

McGee, D.L., 1973, Gulf of Alaska petroleum seeps: Alaska Division of Geological \& Geophysical Surveys, Alaska Open File Report 32, 9 p.

McLean, Hugh, 1977, Organic geochemistry, lithology, and paleontology of Tertiary and Mesozoic rocks from wells on the Alaska Peninsula: United States Geological Survey Open-File Report 77-813, 63 p.

McLean, Hugh, 1979, Sandstone petrology: Upper Jurassic Naknek Formation of the Alaska Peninsula and coeval rocks on the Bering Shelf: Journal of Sedimentary Petrology , v. 49, no. 4, p. 1263-1268.

Merritt, R.D., 1986, Coal resources of the Miocene Unga Conglomerate Member, Bear Lake Formation, Unga Island, Alaska Peninsula: Alaska Division of Geological \& Geophysical Surveys, Public Data File 86-69, 33 p.

Merritt, R.D., and McGee, D.L., 1986, Depositional environments and resource potential of Cretaceous coal-bearing strata at Chignik and Herendeen Bay, Alaska Peninsula: Alaska Division of Geological \& Geophysical Surveys, Public Data File 86-72, 44 p.

Reed, B.L. and Lanphere, M.A., 1973, Alaska-Aleutian Range batholith: geochronology, chemistry, and relation to circum-Pacific plutonism: Geological Society of America Bulletin 84, p. 2583-2610.

Reed, B.L., Miesch, A.T., and Lanphere, M.A., 1983, Plutonic rocks of Jurassic age in the Alaska-Aleutian Range batholith: chemical variations and polarity: Geological Society of American Bulletin, v. 94, p. 1232-1240.

Reifenstuhl, R.R., Shafer, D.C., Ryherd, T.J., Brizzolara, D.W., and Blodgett R.B., 2004, Summary of May 25-June 4, 2004 field notes and samples, Puale Bay and Wide Bay areas, Alaska Peninsula: Alaska Division of Geological \& Geophysical Surveys Raw Data File 2004-3, 16 p.

Silberling, N.J., 1985, Biogeographic significance of the Upper Triassic bivalve Monotis in circum-Pacific accreted terranes, in Howell, D.G., ed., Tectonostratigraphic Terranes of the Circum-Pacific Region: Circum-Pacific Council for Energy and Mineral Resources Earth Sciences Series No. 1, p. 63-70.

Smith, W.R., 1925, The Cold Bay-Katmai district: U.S. Geological Survey Bulletin 773-D, p. 183-207.

Smith, W.R., 1926, Geology and oil developments of the Cold Bay district, Alaska: U.S. Geological Survey Bulletin 783-C, p. 63-88.

Smith, W.R., and Baker, A.A., 1924, The Cold Bay-Katmai district: U.S. Geological Survey Bulletin 755-D, p. 151-218.

Stanton, T.W., and Martin, G.C., 1905, Mesozoic section on Cook Inlet and Alaska Peninsula: Geological Society of America Bulletin, v. 16, p. 391-410.

Triplehorn, D.M., 1976, Large kaolinite crystals in the Chignik Formation (Upper Cretaceous), Herendeen Bay, in Short Notes on Alaskan Geology — 1976: Alaska Division of Geological \& Geophysical Surveys, Geologic Report 51F, p. 23-25.

Wang, Jason, 1986, Sedimentology of Norian (Late Triassic) cherts and carbonates from the Peninsular terrane: Puale Bay, Alaska Peninsula: Syracuse, New York, Syracuse University, unpublished M.S. thesis, 124 p.

Wang, Jason, Newton, C.R., and Dunne, Lorie, 1988, Late Triassic transition from biogenic to arc sedimentation on the Peninsula terrane, Puale Bay, Alaska Peninsula: Geological Society of America Bulletin, v. 100, p. 14661478 . 